Published in final edited form as:

Sci Signal. ; 7(332): pe15. doi:10.1126/scisignal.2005560.

\title{
A Unified Nomenclature and Amino Acid Numbering for Human PTEN
}

Rafael Pulido ${ }^{1,2,{ }^{*},}$ Suzanne J. Baker ${ }^{3}$, Joao T. Barata ${ }^{4}$, Arkaitz Carracedo ${ }^{1,5}$, Victor J. Cid ${ }^{6}$, lan D. Chin-Sang ${ }^{7}$, Vrushank Davé ${ }^{8}$, Jeroen den Hertog ${ }^{9}$, Peter Devreotes ${ }^{10}$, Britta J. Eickholt $^{11}$, Charis Eng ${ }^{12}$, Frank B. Furnari ${ }^{13}$, Maria-Magdalena Georgescu ${ }^{14}$, Arne Gericke $^{15}$, Benjamin Hopkins ${ }^{16}$, Xeujun Jiang ${ }^{17}$, Seung-Rock Lee ${ }^{18}$, Mathias Lösche ${ }^{19}$, Prerna Malaney $^{8}$, Xavier Matias-Guiu ${ }^{20}$, María Molina ${ }^{6}$, Pier Paolo Pandolfi ${ }^{21}$, Ramon Parsons $^{16}$, Paolo Pinton ${ }^{22}$, Carmen Rivas ${ }^{23}$, Rafael M. Rocha ${ }^{24}$, Manuel S. Rodríguez ${ }^{25}$, Alonzo H. Ross ${ }^{26}$, Manuel Serrano ${ }^{27}$, Vuk Stambolic ${ }^{28}$, Bangyan Stiles ${ }^{29}$, Akira Suzuki ${ }^{30}$, Seong-Seng $\operatorname{Tan}^{31}$, Nicholas K. Tonks ${ }^{32}$, Lloyd C. Trotman ${ }^{32}$, Nicolas Wolff ${ }^{33}$, Rudiger Woscholski ${ }^{34}$, Hong $\mathbf{W u}^{35}$, and Nicholas R. Leslie ${ }^{36}$

${ }^{1}$ Ikerbasque, Basque Foundation for Science, Bilbao, Spain ${ }^{2}$ BioCruces Health Research Institute, Barakaldo, Spain ${ }^{3}$ Department of Developmental Neurobiology, St. Jude Children's Research Hospital, Memphis, USA ${ }^{4}$ Instituto de Medicina Molecular, Faculdade de Medicina da Universidade de Lisboa, Lisbon, Portugal ${ }^{5} \mathrm{CIC}$ bioGUNE, Bizkaia Technology Park, Derio, Spain; Biochemistry and Molecular Biology Department, University of the Basque Country (UPV/EHU), Bilbao, Spain ${ }^{6}$ Departamento de Microbiología II, Facultad de Farmacia, Universidad Complutense de Madrid, Instituto Ramón y Cajal de Investigaciones Sanitarias (IRY-CIS), Madrid, Spain ${ }^{7}$ Department of Biology, Queen's University, Kingston, Ontario, Canada ${ }^{8}$ Morsani College of Medicine, Department of Pathology and Cell Biology, Department of Molecular Oncology, H. Lee Moffitt Cancer Center and Research Institute, University of South Florida, Tampa, FL 33620, USA ${ }^{9}$ Hubrecht Institute-KNAW and University Medical Center Utrecht, Utrecht, Netherlands, and Institute of Biology, Leiden, Leiden, Netherlands ${ }^{10}$ Department of Cell Biology, Johns Hopkins University School of Medicine, Baltimore, MD 21205, USA ${ }^{11}$ CharitéUniversitätsmedizin Berlin, Institute of Biochemistry and Cluster of Excellence NeuroCure, Berlin, German ${ }^{12}$ Genomic Medicine Institute, Cleveland Clinic, Cleveland, OH 44195, USA; Department of Genetics and Genome Sciences and CASE Comprehensive Cancer Center, Case Western Reserve University School of Medicine, Cleveland, OH 44106, USA ${ }^{13}$ Ludwig Institute for Cancer Research, University of California San Diego, La Jolla, CA 92093, USA ${ }^{14}$ The University of Texas South-western Medical Center, Dallas, TX 75235, USA ${ }^{15}$ Department of Chemistry and Biochemistry, Worcester Polytechnic Institute, Worcester, MA 01609, USA ${ }^{16}$ Department of Oncological Sciences, Icahn School of Medicine at Mount Sinai, New York, NY 10029, USA ${ }^{17}$ Cell Biology Program, Memorial Sloan Kettering Cancer Center, New York, NY 10065, USA ${ }^{18}$ Department of Biomedical Sciences, Center for Creative Biomedical Scientists, Department of Biochemistry, Research Center for Aging and Geriatrics, Research Institute of Medical Sciences,

Copyright 2014 by the American Association for the Advancement of Science; all rights reserved. Permissions Obtain information about reproducing this article: http://www.sciencemag.org/about/permissions.dtl ${ }^{*}$ Corresponding author. rpulidomurillo@gmail.com. 
Chonnam National University Medical School, Gwangju, Republic of Korea ${ }^{19}$ Physics Department and Department of Biomedical Engineering, Carnegie Mellon University, Pittsburgh. PA 15213, USA; Center for Neutron Research, National Institute of Standards and Technology, Gaithersburg, MD 20899, USA ${ }^{20}$ Department of Pathology and Molecular Genetics/Oncologic Pathology Group, Hospital Universitari Arnau de Vilanova, Lleida, Spain; Biomedical Research Institute of Lleida (IRBLleida), Universitat de Lleida, Lleida, Spain ${ }^{21}$ Cancer Research Institute, Beth Israel Deaconess Cancer Center, Department of Medicine and Pathology, Harvard Medical School, Boston, MA 02115, USA 22Department of Morphology, Surgery and Experimental Medicine, Laboratory for Technologies of Advanced Therapies (LTTA), University of Ferrara, Ferrara, Italy ${ }^{23}$ Departamento de Biología Molecular y Celular, Centro Nacional de BiotecnologíaCSIC, Madrid, Spain; Centro de Investigación en Medicina Molecular y Enfermedades Crónicas, CIMUS, Universidade de Santiago de Compostela (USC), Instituto de Investigaciones Sanitarias (IDIS), Santiago de Compostela, Spain ${ }^{24}$ Research Center, Antonio Prudente Foundation, Hospital A.C. Camargo; Department of Anatomic Pathology, Hospital A.C. Camargo, São Paulo, Brazil ${ }^{25}$ Ubiquitylation and Cancer Molecular Biology, Inbiomed, San Sebastián, Spain ${ }^{26}$ Department of Biochemistry and Molecular Pharmacology, University of Massachusetts Medical School, Worcester, MA 01655, USA ${ }^{27}$ Spanish National Cancer Research Center (CNIO), Madrid, Spain ${ }^{28}$ Department of Medical Biophysics, University of Toronto, Princess Margaret Cancer Center, University Health Network, Toronto, Ontario, Canada ${ }^{29}$ Pharmacology and Pharmaceutical Sciences, USC School of Pharmacy, University of Southern California, Los Angeles, CA 90089, USA ${ }^{30} \mathrm{Global}$ Centers of Excellence Program, Akita University Graduate School of Medicine, Akita, Japan ${ }^{31}$ Brain Development and Regeneration Division, Florey Neuroscience Institutes, The University of Melbourne, Parkville, Victoria, Australia ${ }^{32}$ Cold Spring Harbor Laboratory, Cold Spring Harbor, NY 11724, USA ${ }^{33}$ Institut Pasteur, Unité de Résonance Magnétique Nucléaire des Biomolécules, Département de Biologie Structurale et Chimie, CNRS, Paris, France ${ }^{34}$ Department of Chemistry and Institute of Chemical Biology, Imperial College London, London, UK ${ }^{35}$ Department of Molecular and Medical Pharmacology, University of California, Los Angeles, CA 90095, USA; School of Life Sciences and Peking-Tsinghua Center for Life Sciences, Peking University, Beijing, China ${ }^{36}$ Institute of Biological Chemistry, Biophysics and Bioengineering, School of Engineering and Physical Sciences, Heriot Watt University, Edinburgh, UK

\section{Abstract}

The tumor suppressor PTEN is a major brake for cell transformation, mainly due to its phosphatidylinositol 3,4,5-trisphosphate $\left[\mathrm{PI}(3,4,5) \mathrm{P}_{3}\right]$ phosphatase activity that directly counteracts the oncogenicity of phosphoinositide 3-kinase (PI3K). PTEN mutations are frequent in tumors and in the germ line of patients with tumor predisposition or with neurological or cognitive disorders, which makes the PTEN gene and protein a major focus of interest in current biomedical research. After almost two decades of intense investigation on the 403-residue-long PTEN protein, a previously uncharacterized form of PTEN has been discovered that contains 173 amino-terminal extra amino acids, as a result of an alternate translation initiation site. To facilitate research in the field and to avoid ambiguities in the naming and identification of PTEN amino acids from 
publications and databases, we propose here a unifying nomenclature and amino acid numbering for this longer form of PTEN.

Since the discovery in 1997 of a major tumor suppressor gene encoding a protein with tyrosine phosphatase activity — which was named PTEN (phosphatase and tensin homolog on chromosome ten), MMAC1, or TEP1 (1-3) —an outburst of publications have documented the relevance of PTEN (currently used protein name; official gene name PTEN) on tumor biology and human disease (4). The human PTEN gene is located at chromosome 10q23, a locus frequently deleted in human cancers. In addition, PTEN is a common target of point mutations in tumors, including mutations at noncoding and nontranslated regions, as well as frameshift, missense, and nonsense mutations at coding regions. Patients with PHTS (PTEN hamartoma tumor syndrome), as well as a fraction of patients with ASD (autism spectrum disorders), carry germline PTEN mutations. In the case of PHTS patients, this confers high risk for several types of cancer, including (but not restricted to) breast and thyroid cancer (5-7). PTEN mutations at coding regions distribute all along the gene, and mutations are common in exons encoding the protein tyrosine phosphatase (PTP) catalytic domain, especially exon 5 (8). Although a large number of PTEN mutations found in tumors or in PHTS patients confer total loss of function to the protein, many mutations lead to partial loss of function or have a weak effect on PTEN phosphatase activity. Moreover, most of the germline PTEN mutations from ASD patients do not abrogate PTEN catalysis (9). This makes important not only identifying the PTEN mutation affecting the patient but also characterization of the functional properties of the corresponding mutated PTEN protein.

PTEN is one of relatively few genes in the human genome that encodes two proteins by noncanonical alternative initiation of translation (Fig. 1A). The shorter and more abundant PTEN protein contains 403 amino acids that distribute in two major domains: a catalytic PTP domain and a membrane-binding C2 domain (10). The recently identified and less abundant longer PTEN protein (named as PTEN-Long or PTEN $\alpha$, and here as PTEN-L) contains 173 additional amino-terminal intrinsically disordered amino acids, as a result of the usage of an alternative CUG translation initiation site upstream to the canonical AUG sequence used to produce the shorter 403-amino-acid form (11-13).

Different groups have proposed that PTEN-L can be secreted to enter other cells (11) and that it may form heterodimers with PTEN and regulate mitochondrial function (12). Adding to the functional complexity, PTEN also homodimerizes, which may be particularly important in tumors or patients coexpressing wild-type and mutated PTEN alleles (14). Mutations encoding residues in the specific region of PTEN-L occur in tumors or are reported as polymorphisms (15-19), and this region may control PTEN sub-cellular localization and tumor suppressor activity. For example, this region includes the internalization signal for uptake of PTEN-L into acceptor cells, a postulated physiologic mechanism for tumor suppression, which potentially could be used as a novel therapeutic approach to reconstitute PTEN activity in PTEN-deficient tumors $(11,20)$.

Abundant literature exists using the amino acid numbering from the short PTEN form, but this numbering does not fit with the amino acid numbering of PTEN-L. In addition, the numbering of the specific residues from PTEN-L (1 to 173) is already used to number 
different residues in PTEN, which could generate confusion. For instance, residues 1 to 22 from PTEN-L form part of a predicted secretion signal peptide, whereas residues 6 to 32 from PTEN contain an overlapping $\mathrm{PI}(4,5) \mathrm{P}_{2}$-binding motif, nuclear localization signal, and cytoplasmic localization signal (Fig. 1A) (21-23). Thus, we propose a unified numbering to designate amino acids in PTEN and PTEN-L, so as to avoid ambiguity in the identification of PTEN residues from mutated samples or in the precise naming of PTEN residues in experimental work (Fig. 1, B and C). Our proposal is as follows:

- PTEN-Long is named PTEN-L.

- The amino acid numbering of PTEN does not change.

- The amino acid numbering of PTEN-L is followed by -L, for example, Leu'-L, $\mathrm{Glu}^{2}-\mathrm{L} \ldots$ in three-letter code or L1-L, E2-L ... in single-letter code up to $\mathrm{Val}^{576}$-L or V576-L. Residues Leu ${ }^{1}-\mathrm{L}$ to $\mathrm{Ser}^{22}$-L form part of a predicted secretion signal and would not be present in a mature secreted form of PTEN-L protein.

- The equivalence between residues from PTEN and PTEN-L is calculated by adding 173 to-or subtracting it from-the corresponding numbering. For instance, Cys ${ }^{124}$ (C124) from PTEN is equivalent to Cys ${ }^{297}$-L (C297-L) from PTEN-L.

- Amino acid changes, either through site-directed mutagenesis or through naturally occurring mutations, are indicated as the residue and number without any extension for PTEN (for example, C124S), and with the -L extension for PTEN-L (for example, C297S-L) when the name of the protein is not immediately preceding the mutation name. Mutations commonly used in experimental work to abrogate the catalytic activity of PTEN are shown in Fig. 1B.

- Nucleotide numbering to desig-nate mutations at the PTEN-L-specific residues follows the Human Genome Variation Society (HGVS) recommendations (24). For instance, nucleotides -3 to -1 would encode Asp ${ }^{173}$-L; -6 to -4 would encode Pro ${ }^{172}$-L; up to -519 to -517 , which would encode Leu'1-L (Fig. 1C). Note that CUG -519 to -517 nucleotides in the HGVS-recommended numbering for human PTEN gene correspond to CUG 513 to 515 nucleotides in the human PTEN cDNA entry (NM_000314).

- Homodimers and heterodimers of PTEN proteins are designated with the appropriate extension as needed (Fig. 1D).

- Newly identified PTEN proteins with starting amino acids distinct from $\operatorname{Met}^{1}$ from PTEN or Leu ${ }^{1}$-L from PTEN-L could be named alphabetically as PTEN-M, PTEN$\mathrm{N}$, and so on, or by using another appropriate capital letter, and the nomenclature for amino acids and amino acid changes would follow the rules as above for PTENL.

- The same rules apply to other mammalian PTEN-L protein orthologs, especially those from animal models usually handled in biomedical research.

The possibility of numbering PTEN-L- specific residues with negative numbers starting at and going upstream from the canonical AUG initiation codon of PTEN (as recommended by the HGVS for mutations that introduce in proteins new translation initiation sites) is not 
practical in the case of PTEN-L, because this form is produced from a natural, not mutationcreated, upstream alternative translation initiation codon (CUG) that generates a natural longer protein. The mutations affecting the PTEN-L-specific residues do not introduce new translation initiation sites, but rather change residues in PTEN-L.

We think that this unified nomenclature will facilitate to both researchers and clinicians the unambiguous identification of amino acids from PTEN and PTEN-L and aid in the description of any new forms that may be identified in the future.

\section{References}

1. Li DM, Sun H. TEP1, encoded by a candidate tumor suppressor locus, is a novel protein tyrosine phosphatase regulated by transforming growth factor beta. Cancer Res. 1997; 57:2124-2129. [PubMed: 9187108]

2. Li J, Yen C, Liaw D, Podsypanina K, Bose S, Wang SI, Puc J, Miliaresis C, Rodgers L, McCombie R, Bigner SH, Giovanella BC, Ittmann M, Tycko B, Hibshoosh H, Wigler MH, Parsons R. PTEN, a putative protein tyrosine phosphatase gene mutated in human brain, breast, and prostate cancer. Science. 1997; 275:1943-1947. [PubMed: 9072974]

3. Steck PA, Pershouse MA, Jasser SA, Yung WK, Lin H, Ligon AH, Langford LA, Baumgard ML, Hattier T, Davis T, Frye C, Hu R, Swedlund B, Teng DH, Tavtigian SV. Identification of a candidate tumour suppressor gene, MMAC1, at chromosome 10q23.3 that is mutated in multiple advanced cancers. Nat. Genet. 1997; 15:356-362. [PubMed: 9090379]

4. Song MS, Salmena L, Pandolfi PP. The functions and regulation of the PTEN tumour suppressor. Nat. Rev. Mol. Cell Biol. 2012; 13:283-296. [PubMed: 22473468]

5. Hollander MC, Blumenthal GM, Dennis PA. PTEN loss in the continuum of common cancers, rare syndromes and mouse models. Nat. Rev. Cancer. 2011; 11:289-301. [PubMed: 21430697]

6. Mester J, Eng C. When overgrowth bumps into cancer: The PTEN-opathies. Am. J. Med. Genet. C. Semin. Med. Genet. 2013; 163:114-121. [PubMed: 23613428]

7. Zhou J, Parada LF. PTEN signaling in autism spectrum disorders. Curr. Opin. Neurobiol. 2012; 22:873-879. [PubMed: 22664040]

8. COSMIC (Catalogue of Somatic Mutations in Cancer). http://cancer.sanger.ac.uk/cancergenome/ projects/cosmic/.

9. Rodríguez-Escudero I, Oliver MD, Andrés-Pons A, Molina M, Cid VJ, Pulido R. A comprehensive functional analysis of PTEN mutations: Implications in tumor- and autism-related syndromes. Hum. Mol. Genet. 2011; 20:4132-4142. [PubMed: 21828076]

10. Lee JO, Yang H, Georgescu MM, Di Cristo-fano A, Maehama T, Shi Y, Dixon JE, Pandolfi P, Pavletich NP. Crystal structure of the PTEN tumor suppressor: Implications for its phosphoinositide phosphatase activity and membrane association. Cell. 1999; 99:323-334. [PubMed: 10555148]

11. Hopkins BD, Fine B, Steinbach N, Dendy M, Rapp Z, Shaw J, Pappas K, Yu JS, Hodakoski C, Mense S, Klein J, Pegno S, Sulis ML, Goldstein H, Amendolara B, Lei L, Maurer M, Bruce J, Canoll P, Hibshoosh H, Parsons R. A secreted PTEN phosphatase that enters cells to alter signaling and survival. Science. 2013; 341:399-402. [PubMed: 23744781]

12. Liang H, He S, Yang J, Jia X, Wang P, Chen X, Zhang Z, Zou X, McNutt MA, Shen WH, Yin Y. PTENá, a PTEN isoform translated through alternative initiation, regulates mitochondrial function and energy metabolism. Cell Metab. 2014; 19:836-848. [PubMed: 24768297]

13. Malaney P, Uversky VN, Davé V. The PTEN Long N-tail is intrinsically disordered: Increased viability for PTEN therapy. Mol. Biosyst. 2013; 9:2877-2888. [PubMed: 24056727]

14. Papa A, Wan L, Bonora M, Salmena L, Song MS, Hobbs RM, Lunardi A, Webster K, Ng C, Newton RH, Knoblauch N, Guarnerio J, Ito K, Turka LA, Beck AH, Pinton P, Bronson RT, Wei W, Pandolfi PP. Cancer-associated PTEN mutants act in a dominant-negative manner to suppress PTEN protein function. Cell. 2014; 157:595-610. [PubMed: 24766807] 
15. Ishihara H, Sasaoka T, Kagawa S, Murakami S, Fukui K, Kawagishi Y, Yamazaki K, Sato A, Iwata M, Urakaze M, Ishiki M, Wada T, Yaguchi S, Tsuneki H, Kimura I, Kobayashi M.

Association of the polymorphisms in the 5'-untranslated region of PTEN gene with type 2 diabetes in a Japanese population. FEBS Lett. 2003; 554:450-454. [PubMed: 14623110]

16. Poetsch M, Dittberner T, Woenckhaus C. PTEN/MMAC1 in malignant melanoma and its importance for tumor progression. Cancer Genet. Cytogenet. 2001; 125:21-26. [PubMed: 11297763]

17. Poetsch M, Lorenz G, Kleist B. Detection of new PTEN/MMAC1 mutations in head and neck squamous cell carcinomas with loss of chromosome 10. Cancer Genet. Cytogenet. 2002; 132:2024. [PubMed: 11801303]

18. Sakai A, Thieblemont C, Wellmann A, Jaffe ES, Raffeld M. PTEN gene alterations in lymphoid neoplasms. Blood. 1998; 92:3410-3415. [PubMed: 9787181]

19. Tunca B, Bekar A, Cecener G, Egeli U, Vatan O, Tolunay S, Kocaeli H, Aksoy K. Impact of novel PTEN mutations in Turkish patients with glioblastoma multiforme. J. Neurooncol. 2007; 82:263269. [PubMed: 17151929]

20. Leslie NR, Brunton VG. Cell biology. Where is PTEN? Science. 2013; 341:355-356. [PubMed: 23888029]

21. Denning G, Jean-Joseph B, Prince C, Durden DL, Vogt PK. A short N-terminal sequence of PTEN controls cytoplasmic localization and is required for suppression of cell growth. Oncogene. 2007; 26:3930-3940. [PubMed: 17213812]

22. Gil A, Andrés-Pons A, Fernández E, Valiente M, Torres J, Cervera J, Pulido R. Nuclear localization of PTEN by a Ran-dependent mechanism enhances apoptosis: Involvement of an Nterminal nuclear localization domain and multiple nuclear exclusion motifs. Mol. Biol. Cell. 2006; 17:4002-4013. [PubMed: 16807353]

23. Walker SM, Leslie NR, Perera NM, Batty IH, Downes CP. The tumour-suppressor function of PTEN requires an N-terminal lipid-binding motif. Biochem. J. 2004; 379:301-307. [PubMed: 14711368]

24. HGVS. www.hgvs.org/mutnomen/recs-prot.html.

25. Davidson L, Maccario H, Perera NM, Yang X, Spinelli L, Tibarewal P, Glancy B, Gray A, Weijer CJ, Downes CP, Leslie NR. Suppression of cellular proliferation and invasion by the concerted lipid and protein phosphatase activities of PTEN. Oncogene. 2010; 29:687-697. [PubMed: 19915616]

26. Maehama T, Dixon JE. The tumor suppressor, PTEN/MMAC1, dephosphorylates the lipid second messenger, phosphatidylinositol 3,4,5-trisphosphate. J. Biol. Chem. 1998; 273:13375-13378. [PubMed: 9593664]

27. Myers MP, Pass I, Batty IH, Van der Kaay J, Stolarov JP, Hemmings BA, Wigler MH, Downes CP, Tonks NK. The lipid phosphatase activity of PTEN is critical for its tumor supressor function. Proc. Natl. Acad. Sci. U.S.A. 1998; 95:13513-13518. [PubMed: 9811831] 
A

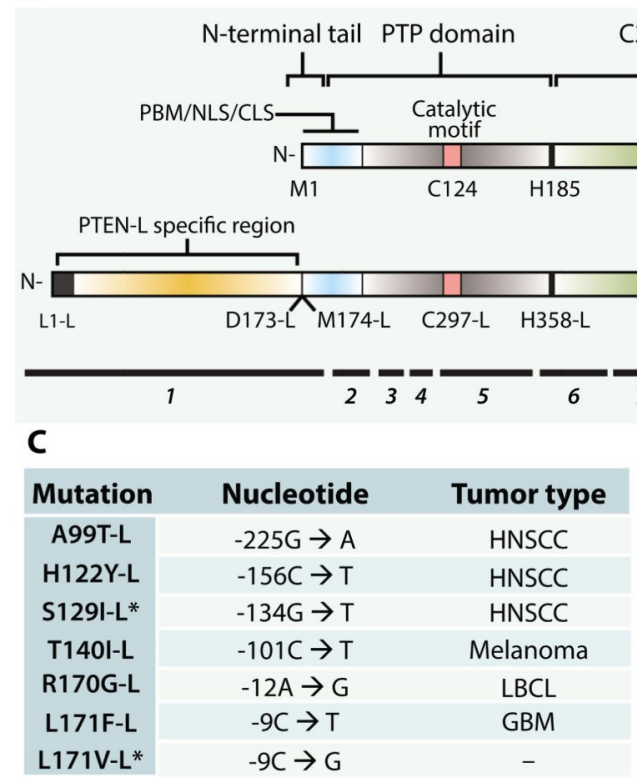

C2 domain C-terminal tail L
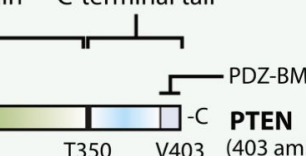

T350 V403 (403 amino acids)

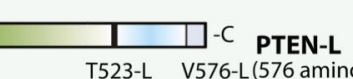

T523-L V576-L(576 amino acids)

8

8

D

\begin{tabular}{|ccc|}
\hline Type of dimer & Dimer nomenclature & Examples \\
\hline PTEN homodimer & PTEN:PTEN & PTEN:PTEN \\
PTEN:PTEN C124S \\
PTEN-L homodimer & PTEN-L:PTEN-L & PTEN-L:PTEN-L \\
$\begin{array}{c}\text { PTEN-L:PTEN-L C297S-L } \\
\text { PTEN:PTEN-L } \\
\text { heterodimer }\end{array}$ & PTEN:PTEN-L & PTEN:PTEN-L \\
& & PTEN C124S:PTEN-L \\
\hline
\end{tabular}

Fig. 1. A nomenclature for PTEN-L amino acid numbering

(A) Schematic of human PTEN and PTEN-L and the proposed numbering of amino acids. The domains common to PTEN and PTEN-L are indicated at the top, and the amino acids flanking the domains are indicated below each protein depiction. C124 (PTEN) or C297-L (PTEN-L) corresponds to the catalytic Cys. The N- and C-terminal tails from PTEN, and the PTEN-L-specific region (residues L1-L to D173-L) are intrinsically disordered regions. The black box at the $\mathrm{N}$ terminus of PTEN-L corresponds to a predicted secretion signal (predicted cleavage site at amino acid S22-L). PBM, PI(4,5) $\mathrm{P}_{2}$-binding motif; NLS, nuclear localization sequence; CLS, cytoplasmic localization sequence; PDZ-BM, PDZ-binding motif. Numbers at the bottom correspond to exon numbering. (B) Examples of nomenclature for commonly used PTEN mutations totally or partially defective for phosphatase activity (25-27). (C) Examples of nomenclature for identified PTEN mutations targeting PTEN-Lspecific amino acids. Loss of function has been experimentally observed for mutations A99T-L, H122Y-L, and R170G-L [(11); note that in reference (11) the amino acid numbering is one unit less]. *Reported as polymorphisms $(15,17)$. HNSCC, head and neck squamous cell carcinoma; LBCL, large B cell lymphoma; GBM, glioblastoma multiforme.

(D) Examples of nomenclature for PTEN dimers. Examples are provided of different combinations of PTEN and PTEN-L wild-type and mutated homodimers and heterodimers. PTEN:PTEN homodimers (14) and PTEN:PTEN-L heterodimers (12) have been demonstrated experimentally. The possibility also exists of dimers containing two mutated proteins (with the same mutation or different mutations). 